\title{
Production of a-cuprenene in Xanthophyllomyces dendrorhous: a step closer to a potent terpene biofactory
}

\author{
Elena Melillo ${ }^{1}$, Rita Setroikromo ${ }^{1}$, Wim J Quax ${ }^{1^{*}}$ and Oliver Kayser ${ }^{2}$
}

\begin{abstract}
Background: The red yeast Xanthophyllomyces dendrorhous is a natural producer of the carotenoid astaxanthin. Because of its high flux, the native terpene pathway leading to the production of the tetraterpene is of particular interest as it can be redirected toward the production of other terpene compounds. The genetic tools for the transformation of the yeast with the concurrent knock-out of genes involved in the astaxanthin biosynthesis are made available and here we show that the production of the sesquiterpene a-cuprenene is possible in mutant strains of $X$. dendrorhous transformed with the Cop6 gene originating from the fungus Coprinus cinereus. For the evaluation of the production levels, we chose to express the same gene and analyze the accumulation of acuprenene in Escherichia coli and Saccharomyces cerevisiae, as well. Here we propose that $X$. dendrorhous is a candidate in the search for the potential platform organism for the production of terpenes.

Results: All three $X$. dendrorhous mutants functionally express the Cop6 gene and accumulate a-cuprenene. The production of a-cuprenene in the red yeast reached $80 \mathrm{mg} / \mathrm{L}$, which represents a far higher concentration compared to the levels obtained in the E. coli and S. cerevisiae mutants. At this expression levels the pool of terpene precursors has not become a limiting factor in the $X$. dendrorhous mutants since the expression of the Cop6 gene in the genomic rDNA of the yeast allows production of both a-cuprenene and astaxanthin without affecting the growth or the accumulation levels of both compounds.

Conclusions: We have shown that $X$. dendrorhous can produce a-cuprenene, and the results here presented, next to the capability of accumulating at least two more non-native sesquiterpenes, demonstrates the high potential of this yeast to become an interesting terpene-based drugs producer.
\end{abstract}

Keywords: Xanthophyllomyces dendrorhous, a-cuprenene, Metabolic engineering, Escherichia coli, Saccharomyces cerevisiae, Terpene cell factory

\section{Background}

Since ancient times, microorganisms have been used to produce bread, wine and dairy products in order to improve the quality of food and its nutrients. Today, microbes are utilized for the manufacture of a wide variety of fine or bulk chemicals including antibiotics [1,2], vitamins [3], biofuels [4,5], biodegradable and biocompatible plastics from waste [6] and terpene-based drugs $[7,8]$. Originally, the choice for a production host was

\footnotetext{
* Correspondence: w.j.quax@rug.nl

'Department of Pharmaceutical Biology, Groningen Research Institute of Pharmacy, University of Groningen, A. Deusinglaan 1, Groningen 9713AV, The Netherlands

Full list of author information is available at the end of the article
}

dictated by the ability of the organism to produce the desired compounds, but in most cases the concentration of the chemicals of interest was not sufficient to cover the market demand.

With the development of genetic engineering, new tools became available to overcome these obstacles. The possibility of transferring single genes or even complete pathways to other microorganisms led to improved yields or easier bioprocessing conditions. Furthermore, metabolic engineering allowed the enhancement of the yields in the native hosts by finely tuning the metabolic networks of the cells towards the optimized production of the specific compound. More recently, the combination of synthetic biology and metabolic engineering

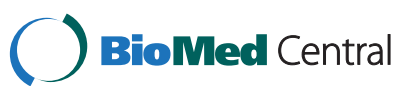


has resulted in the creation of hosts capable of producing a non-native compound with high efficiency obtainable only by optimizing the native pathways of the cells [9].

The majority of the yeast or bacterial strains used by industry have been selected for the several advantages they deliver: they are easy to cultivate, they can grow on cheap media, they are generally regarded as safe (GRAS status) and their metabolic pathways are easy to modify via genetic engineering.

Xanthophyllomyces dendrorhous, a red basidiomycetous yeast, represents one of the microbial strains already used in industry and shares all the aforementioned advantages [10]. Today, X. dendrorhous is grown at industrial scale for its native capability to produce the valuable carotenoid astaxanthin.

Carotenoids, together with several other pharmaceutically important compounds, like artemisinin and Taxol, belong to the natural compounds class of the terpenes [11]. Several efforts have been put in the engineering of a platform organism for the production of industrially important terpenes $[7,8]$.

We hypothesize that, since $X$. dendrorhous can produce high levels of astaxanthin, which shares the same precursors with all other terpenes, it can also utilize those same precursors for the production of any other terpenoid compound.

The red yeast was already shown to be able to functionally express the pentalenene synthase from a Streptomyces strain involved in the biosynthesis of the antibiotic pentalenolactone [12]. In order to further evaluate the potential of the red yeast as a platform organism for terpenes, we expressed the sesquiterpene cyclase Cop6 in X. dendrorhous mutant strains. The protein Cop6, originating from the fungus Coprinus cinereus, produces the cyclized sesquiterpene $\alpha$-cuprenene, which is the basic structure for the formation of lagopodin A, an antimicrobial sesquiterpene quinone [13]. We also compared the accumulation levels of $\alpha$-cuprenene with two of the most industrially utilized microbial strains, E. coli and S. cerevisiae.

\section{Results}

\section{Production of cuprenene in E. coli}

Isolation of volatile terpenoids in $E$. coli via addition of a dodecane organic phase to the liquid cultures has been shown to be extremely efficient [14]. We have decided to adopt the same strategy to capture the $\alpha$-cuprenene produced by the $E$. coli strains transformed with the Cop6 gene. In order to be able to compare the levels of $\alpha$-cuprenene at the different time points and from different organisms we added hexadecane in known concentrations, as an internal standard, to the dodecane.

The wild type strain and the transformed one exhibited the same growth curve and biomass accumulation; twenty hours after induction of the expression, the cells reached the highest density and then started dying after 30 hours. After 48 hours the concentration of cuprenene, based on the internal standard, in the modified strain reached approximately $0.25 \mathrm{mg} / \mathrm{L}$ of culture, a slight increase compared to the concentration at 20 hours (Figure 1).

\section{Growth curves and a-cuprenene production from}

\section{$S$. cerevisiae and $X$. dendrorhous in rich medium}

After the separate transformations of $X$. dendrorhous wild-type strain with the vectors pCrtE-Cop6, pCrtYBCop6 and pPR-Cop6, one colony from each transformation plate was chosen to be grown and analyzed. As expected, since the astaxanthin pathway was disrupted (Figure 2), on the plates used to select $\Delta E$-Cop6 and $\triangle Y B$-Cop6, the mutant colonies presented a white phenotype. In contrast, XdCop 6 colonies, transformed with pPR-Cop6, in which the carotenoid pathway was not modified (Figure 2), shared an orange pigmentation with the wild type strain.

The S. cerevisiae mutant, ScCop6, was isolated after transformation of the wild type strain of $S$. cerevisiae with the plasmid p426GPD-Cop6, which allows constitutive expression of the Cop6 gene.

A time course analysis was performed on the ScCop6 and on the three $X$. dendrorhous mutant strains, $X d C o p 6, \triangle E$-Cop6 and $\triangle Y B$-Cop6. The strains were grown in YPD medium in order to obtain a high growth in shake flasks. The time course consisted of four sampling times after the inoculation in fresh medium; we chose $24 \mathrm{~h}, 48 \mathrm{~h}, 72 \mathrm{~h}$ and $96 \mathrm{~h}$ to be able to observe all the steps of the growth curve.

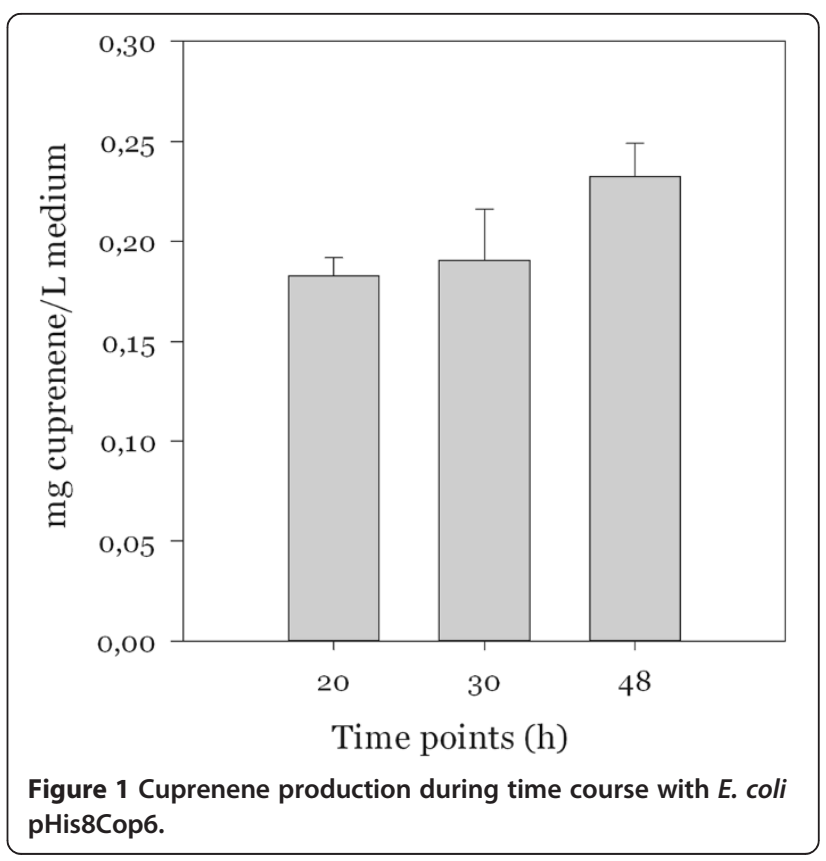



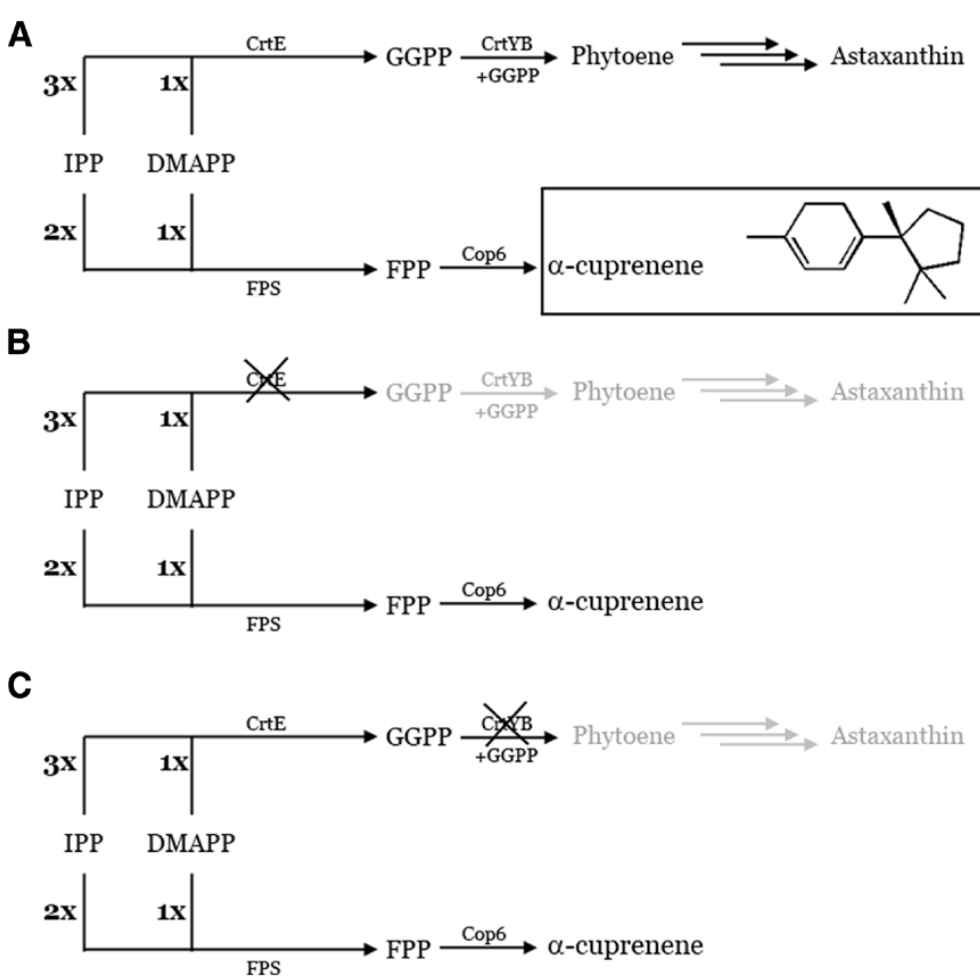

Figure 2 Schematic representation of $X$. dendrorhous mutant strains. (A) In the mutant $X d C o p 6$, the native astaxanthin pathway has not been modified but the gene Cop6 has been integrated in the rDNA of the yeast allowing the mutant to produce both astaxanthin and a-cuprenene. (B) In the strain $\triangle E$-Cop6 the Cop6 gene has been inserted in the crtE gene causing the disruption of the carotenoid production at the GGPP synthesis level. (C) When Cop6 is inserted in the crtYB gene, the $\triangle Y B$-Cop6 strain is created. While there is still expression of the GGPPS, phytoene cannot be produced anymore, blocking the production of astaxanthin one step downstream of the $\Delta E$-Cop6.

With respect to the $\mathrm{OD}_{600}$ and to the cell dry weight, the $S$. cerevisiae and $X$. dendrorhous mutants exhibit similar curves compared to the respective wild type strains.

Figure 3 compares the growth curves obtained during the time course analysis from the mutant strains of both yeasts. In spite of the fact that $S$. cerevisiae cells reach a higher optical density, they accumulate a lower biomass compared to all $X$. dendrorhous strains.

The diluted dodecane solutions from the $S$. cerevisiae strains and from the wild type and mutant $X$. dendrorhous were analyzed by GCMS, and a single peak appeared in the chromatograms from the mutants at 12.8 minutes (Figure 4). The fragmentation pattern of the peak was compared to the pattern corresponding to $\alpha$-cuprenene produced in S. cerevisiae (Figure 5). The mass and the relative ratio of the fragment peaks matched between the two patterns, allowing us to confirm that the only sesquiterpene produced by Cop6 in $X$. dendrorhous is $\alpha$-cuprenene.

The time-course production of $\alpha$-cuprenene in the four strains is represented in Figure 6. The level of the sesquiterpene in S. cerevisiae sharply increased after 24 hours to reach a maximum of $6.6 \mathrm{mg} / \mathrm{L}$ on the second day of culturing and then appeared to decrease during the following two days.
The $\alpha$-cuprenene production in the three $X$. dendrorhous strains showed ten times higher levels with a major increase between 24 and 48 hours, as well. In contrast to $S$. cerevisiae, XdCop6, $\triangle E-C o p 6$ and $\triangle Y B-C o p 6$ constantly produced $\alpha$-cuprenene during the complete time course. The accumulation of the sesquiterpene appeared to be consistent in all the three $X$. dendrorhous strains and was directly proportional to the cell mass, in particular in the first three days after inoculation. The highest yield was obtained after 96 hours with the $X d C o p 6$ strain and corresponded to almost $80 \mathrm{mg} / \mathrm{L}$ of culturing medium. $\Delta E$-Cop 6 could produce up to $70 \mathrm{mg}$ of $\alpha$-cuprenene per liter of medium and $\triangle Y B-C o p 674 \mathrm{mg} / \mathrm{L}$, both after growing for 96 hours.

\section{Growth curves and a-cuprenene production from} $S$. cerevisiae and $X$. dendrorhous in minimal medium

The ScCop6 mutant was selected on medium lacking uracil in order to allow only the colonies containing the plasmid to grow. The rich YPD medium contains all the necessary nucleotides, thus the selective pressure on the $S$. cerevisiae mutant strain grown in this medium was inexistent. For this reason, we decided to grow all the strains in a minimal medium lacking uracil which would 


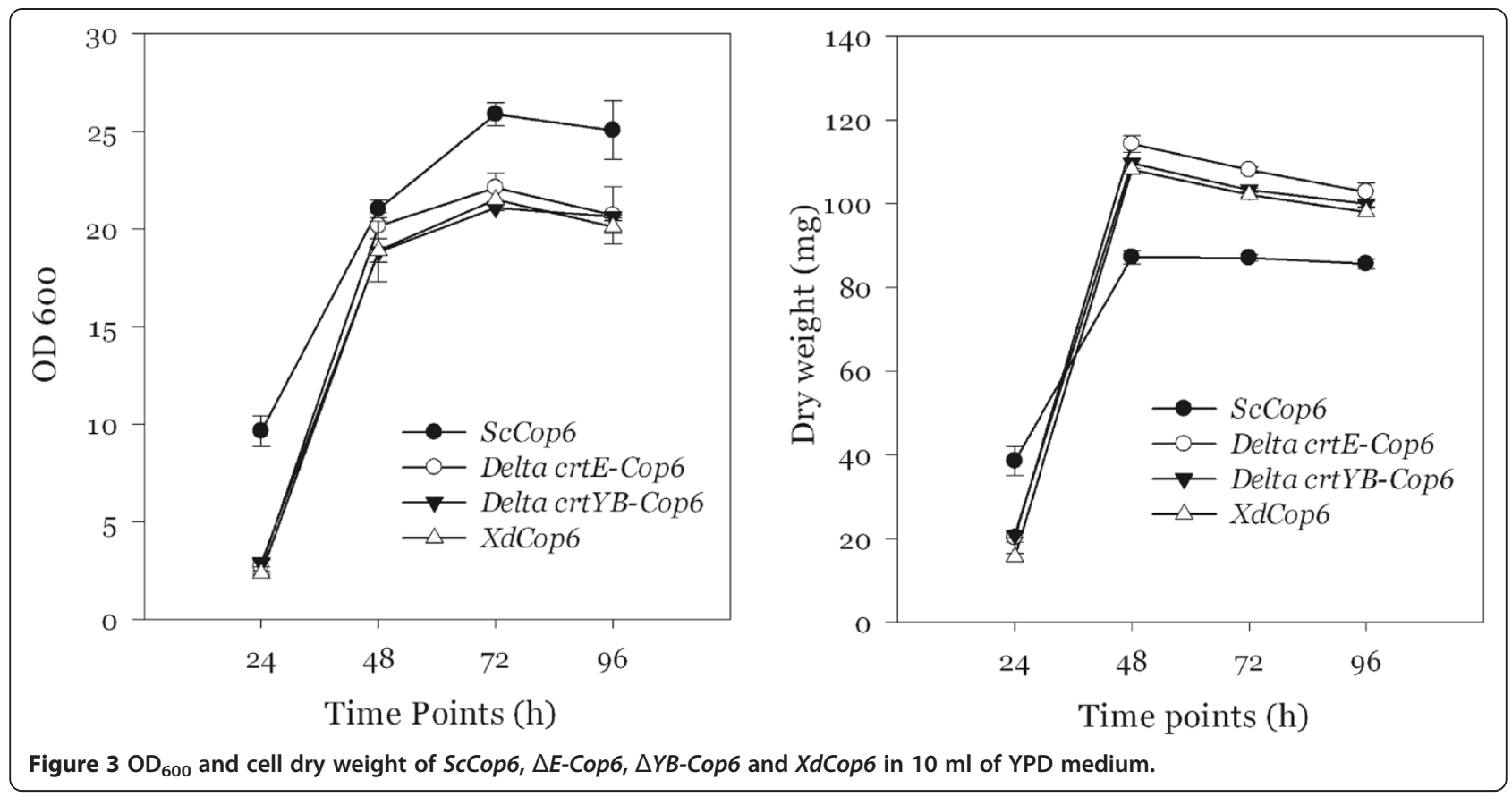

allow a more accurate comparison between the ScCop6 and the three $X$. dendrorhous mutants. The same settings chosen for the time course in rich medium were applied for the growth and $\alpha$-cuprenene production analysis in minimal medium.

An overall lower cell mass and $\mathrm{OD}_{600}$ compared to rich medium was observed for all the strains and no difference in growth could be discerned between the wild type strains and the mutant strains, both for S. cerevisiae and $X$. dendrorhous.

S. cerevisiae strains started growing already after 24 hours while $X d C o p 6, \triangle E$-Cop6 and $\triangle Y B$-Cop6 showed a lag phase between 0 and 24 hours and a log growth between 24 and 48 hours (Figure 7). In minimal medium $\Delta E$-Cop6 exhibited a slightly reduced cell growth compared to $X d$ Cop 6 and $\triangle Y B-C o p 6$.

The accumulation of $\alpha$-cuprenene in the three $X$. dendrorhous strains was significantly lower than the concentration obtained in YPD reaching a maximum of $20 \mathrm{mg} / \mathrm{l}$ (Figure 8) for $\triangle Y B-C o p 6$. When ScCop6 was grown in minimal selective medium, the sesquiterpene production increased nearly to $12 \mathrm{mg}$ of $\alpha$-cuprenene per liter of medium.

\section{Microscopy analysis of ScCop6 and XdCop6}

Figures 3 and 7 indicate that $S$. cerevisiae and $X$. dendrorhous are characterized by a different growth rate and optical density. For a better understanding of the
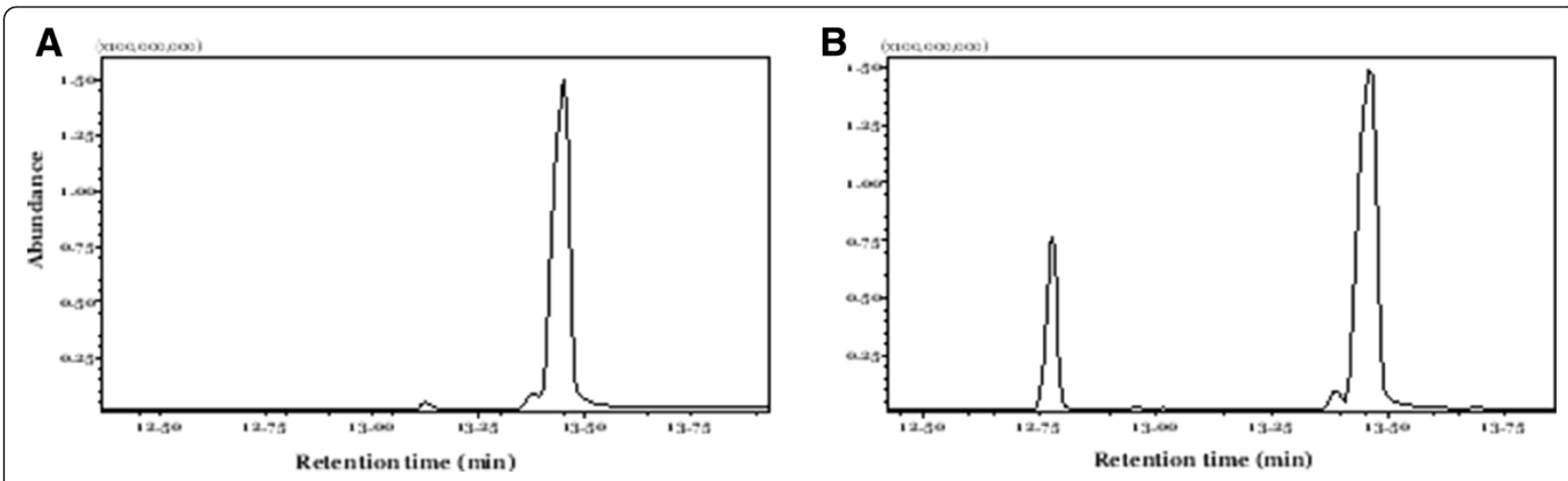

Figure 4 Chromatograms of the diluted dodecane from (A) X. dendrorhous wild type strain and (B) $\Delta E$-Cop6. The peak at 13.5 minutes is the hexadecane used as internal standard. The a-cuprenene has a retention time of approximately 12.8 minutes. 


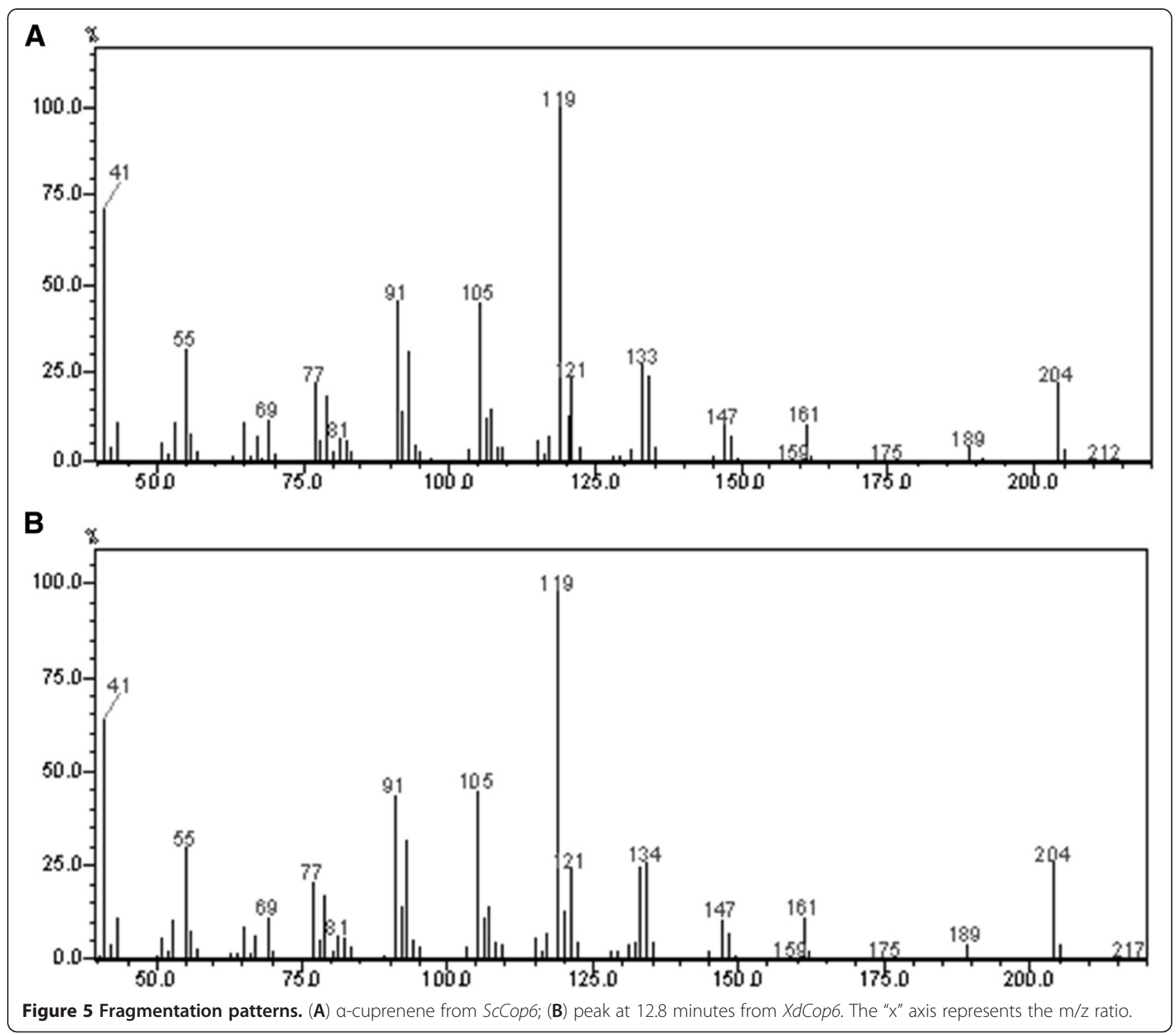

differences between the two yeasts, we performed a microscopy analysis on the strains ScCop6 and XdCop6.

A Burke chamber was used to count the yeast cells. In spite of the macroscopic red pigmentation of XdCop6, the cells appeared white under the microscope white light. After counting samples in duplo from ScCop6 and $X d C o p 6$ and calculating the cell numbers, we observed that one $\mathrm{OD}_{600}$ unit in $S$. cerevisiae corresponded to $3 \mathrm{x}$ $10^{7}$ cells per milliliter, consistent with the data available in literature [15]. In contrast, the number of $X$. dendrorhous cells counted in one milliliter of culture with the same optical density was $4.8 \times 10^{6}$.

To further ascertain the morphological differences between ScCop6 and XdCop6, we compared the average cells sizes. Figure 9 compares the pictures of the two strains after four days of growth; the same magnification was used to visualize the cells. Cells from XdCop6 are round shaped and have a granular appearance, while ScCop6 cells, although showing a similar round morphology, show a more homogeneous cytosol. On average, the size of $X$. dendrorhous cells was $10 \mu \mathrm{m}$ in diameter; $S$. cerevisiae cells were smaller with a size ranging between 7 and $8 \mu \mathrm{m}$. Not only ScCop6 cells were on average smaller than $X$. dendrorhous, but they also never had a diameter bigger than $10 \mu \mathrm{m}$, in contrast with the red yeast cells.

\section{Discussion}

E. coli and S. cerevisiae are two model organisms that also serve as industrial cell factories for the production of a wide variety of compounds ranging from pharmaceutically active substances to food ingredients and biofuels.

In spite of the fact that $X$. dendrorhous has not been studied as extensively as E. coli or S. cerevisiae, it shows 


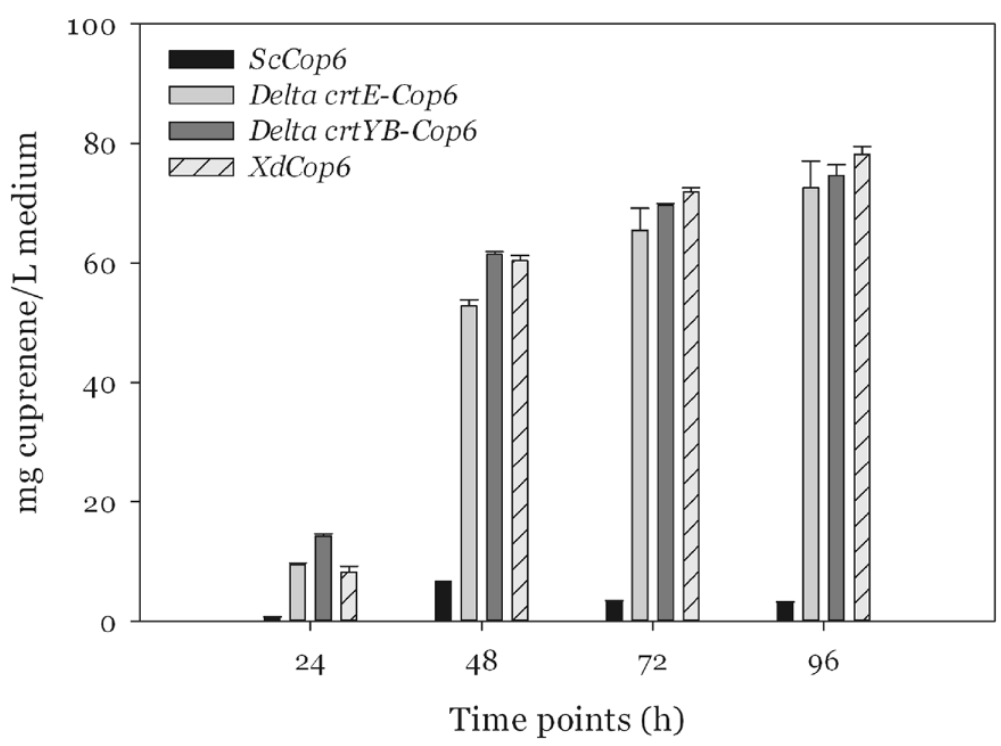

Figure 6 Production of a-cuprenene in rich YPD medium.

great potential to become a platform organism for terpene production [12]. In order to assess the value of the red yeast as a cell factory, we expressed the Cop6 gene in the three $X$. dendrorhous mutants and compared the production of $\alpha$-cuprenene with $E$. coli and S. cerevisiae strains expressing the same gene.

The cDNA from the Cop6 gene was expressed by all $X$. dendrorhous mutants, whereas the genomic version of the gene, when transferred to the red yeast, did not result in $\alpha$-cuprenene accumulation (data not shown) indicating that $X$. dendrorhous cannot correctly splice the gene from $C$. cinereus.

When grown in rich medium, all $X$. dendrorhous strains, including the wild type, showed very similar growth rates: they reached $\mathrm{OD}_{600}$ values of 20 and produced a maximum of 12 grams of cell dry weight per liter of culture. Similarly, when looking at the $\alpha$-cuprenene production, the three strains $X d C o p 6, \triangle E-C o p 6$ and $\triangle Y B$-Cop6 did not show big differences among each other, with levels of the sesquiterpene ranging from 70 to $80 \mathrm{mg}$ of compound per liter of medium.

Comparing Figures 3 and 7, it is clear that, differently from the experiments in the rich medium, the three $X$. dendrorhous strains shared an altered growth behavior in the minimal medium. While XdCop6 and $\triangle Y B$-Cop 6 reached a maximum cell dry mass of nearly $5 \mathrm{~g} / \mathrm{L}$, similar to the one obtained with the wild type strain, $\Delta E$ Cop6 could not produce more than $3.5 \mathrm{~g}$ of dry cells per liter of medium. In 2008 Niklitschek and colleagues have reported the difficulty to isolate a $X$. dendrorhous strain
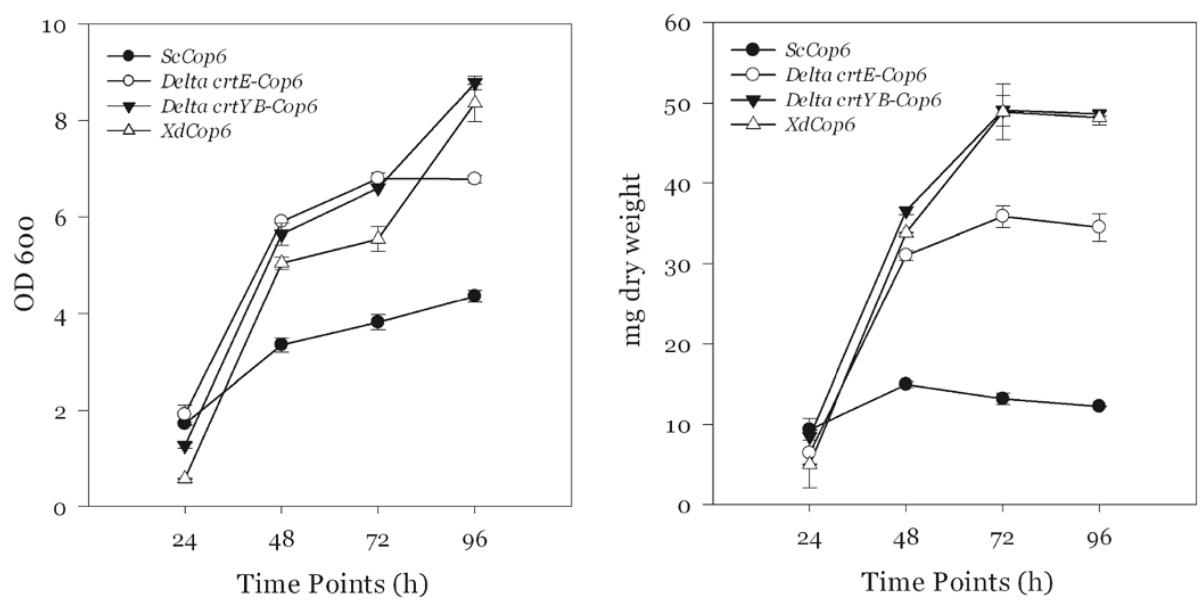

Figure $7 \mathrm{OD}_{600}$ and cell dry weight of ScCop6, $\triangle E$-Cop6, $\triangle Y B$-Cop6 and XdCop6 in $10 \mathrm{ml}$ of minimal medium. 


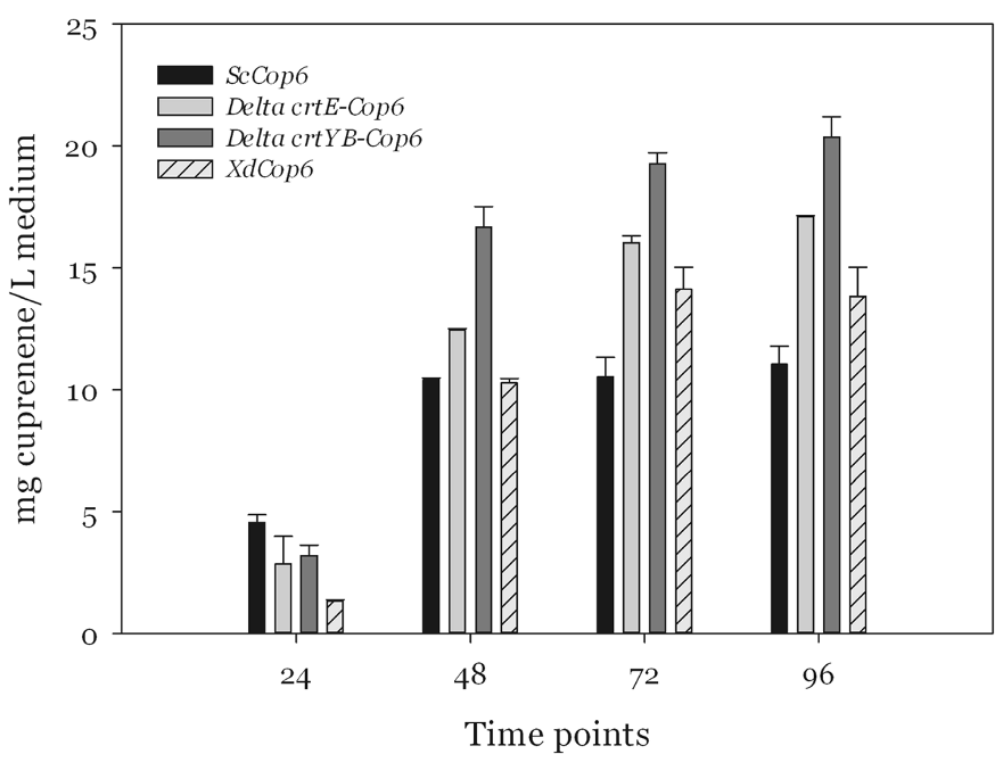

Figure 8 Production of a-cuprenene in minimal medium.

in which both crtE alleles had been knocked out [16], suggesting an important role of this protein in the yeast growth. In the light of the results shown in this study, we can conclude that difference in growth between the $\Delta E$-Cop6 mutant and all the other $X$. dendrorhous strains in the minimal medium could be explained by a lack, in this particular medium, of compounds important for the yeast growth produced directly or indirectly by the $\mathrm{CrtE}$ protein.

Concomitantly with the reduced growth in the minimal medium, the concentration of $\alpha$-cuprenene in the dodecane was also affected, reaching values ranging from 15 to $21 \mathrm{mg} / \mathrm{L}$ of culture. The decrease in sesquiterpene accumulation can partly be explained by the reduced cell mass and partly by the lower concentration of nutrients in the minimal medium which would induce the cells to minimize the energy consumption by shutting down unnecessary pathways.

When comparing cell mass accumulation and $\alpha$-cuprenene production in all $X$. dendrorhous, E. coli and S. cerevisiae strains, the prokaryote showed the lowest values. The low biomass in the bacterium is most likely to be ascribed to a lack of glucose in its growth medium, while the limited sesquiterpene production is due to the lower terpene flux in E. coli compared to the two eukaryotes.

The differences in growth curves and dry weight between the $X$. dendrorhous and the $S$. cerevisiae strains seem to have morphological reasons. $X$. dendrorhous cells are on average bigger than S. cerevisiae ones [17] and at the same optical density $S$. cerevisiae cell counts

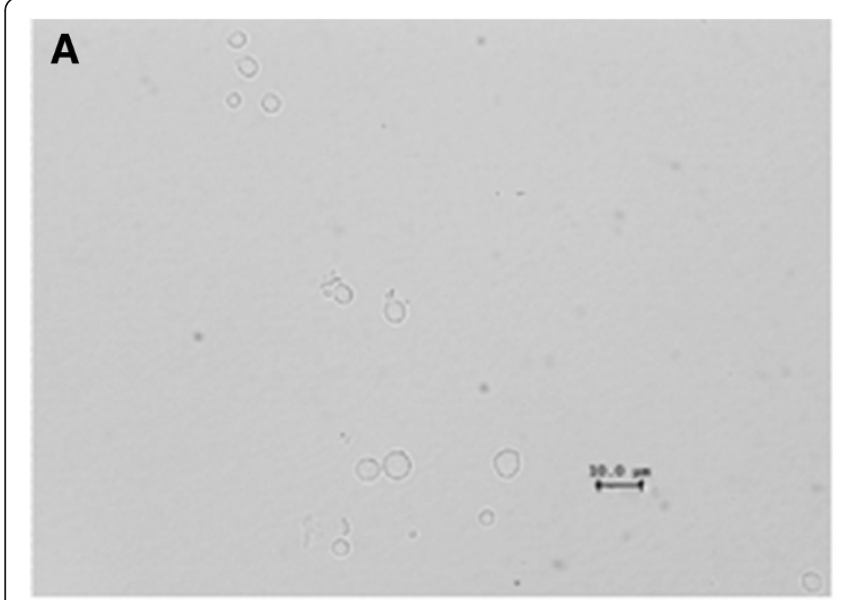

B

Figure 9 Microscopy photographs. (A) ScCop6 and (B) XdCop6. 
are almost 10 times higher than in $X$. dendrorhous cultures, meaning that the same $\mathrm{OD}_{600}$ value corresponds to more $S$. cerevisiae cells than it does for $X$. dendrorhous. Since, at the beginning of the time course experiments, the initial $\mathrm{OD}_{600}$ for all the strains was set at 0.05 , the number of cells initially transferred to the fresh medium was higher in ScCop6 than in all the $X$. dendrorhous mutants. This would explain the delay in growth we observed for XdCop6, $\triangle E$-Cop6 and $\triangle Y B$ Cop6 compared to ScCop6. Additionally, the higher cell mass accumulation observed in the $X$. dendrorhous strains compared to $S$. cerevisiae may be due to the red yeast's bigger sized cells rather than to a higher number of cells.

The highest $\alpha$-cuprenene production levels were obtained with the $X$. dendrorhous strains both in the rich and in the minimal medium experiments. Remarkably, in the YPD medium the gap in sesquiterpene accumulation between the red yeast and the $S$. cerevisiae strain was far more pronounced. We assume that, since the complete medium does not allow selective pressure on ScCop6, which was isolated by its ability to grow on minimal medium lacking uracil, the strain might have undergone a reduction in plasmid copy number.

While ScCop6 mutants contain an average of 20 copies of Cop6, the $X$. dendrorhous white mutants possess just one copy of the gene since the recombination of the constructs can occur only once in the single crtE or $\operatorname{crt} Y B$ genes. In order to obtain a mutant with a higher number of integrations of the gene in the genomic rDNA, we transformed the $X$. dendrorhous wild type strain with a higher concentration of the DNA fragment from the pPR-Cop6 vector and selected the transformants on YPD medium containing a concentration of geneticin 5 times higher than normal, hoping for gene amplification. Unfortunately, no colony grew after this transformation and we could not evaluate the effect of more gene copies on the $\alpha$-cuprenene accumulation.

Nevertheless, we can safely assume that the concentration of the precursors is not a limiting factor in the sesquiterpene production in $X$. dendrorhous, since the strain $X d$ Cop 6 can easily sustain the production of both $\alpha$-cuprenene and astaxanthin, especially when grown in the YPD rich medium. The production of both terpene compounds in XdCop6 confirms the hypothesis that a higher gene copy number would positively influence the $\alpha$-cuprenene production in $X$. dendrorhous.

In conclusion, $X$. dendrorhous shows great promise since it has the GRAS status, it grows at room temperature in minimal media, and it has already been used by industry for the production of astaxanthin. We discovered that it can produce at least three non-native sesquiterpenes, pentalenene [12], $\alpha$-cuprenene and cubebol (data not shown). Furthermore, $X$. dendrorhous is the best microorganism, among the ones we have analyzed, to be used for the production of $\alpha$-cuprenene. A better understanding of the molecular biology of this yeast will prove useful for the identification of stronger promoters for a higher gene expression.

In light of the aforementioned advantages and of the provided results, $X$. dendrorhous is an interesting candidate for being used as a cell factory for the production of terpenes.

\section{Methods}

\section{Strains and culture conditions}

The E. coli strain DH5 $\alpha$ was used for the cloning processes, while the strain BL21 (DE3) was transformed and cultured for the time course experiments. S. cerevisiae MRG 5 \#502 (MATa, ura3-52, leu2- $\Delta 1$, trp1- $\Delta 36$, his3$\Delta 200$, Dade2) was used for the transformation and time course analysis. $X$. dendrorhous wild type strain (CBS 6938) was used for the transformations and as negative control for all experiments. Both $E$. coli strains were grown in LB (10 g/L Trypton, 5 g/L Yeast Extract, $10 \mathrm{~g} / \mathrm{L}$ $\mathrm{NaCl}$ ) with $30 \mathrm{mg} / \mathrm{ml}$ kanamycin. The rich medium for $S$. cerevisiae and $X$. dendrorhous was YPD (10 g/L Yeast Extract, $20 \mathrm{~g} / \mathrm{L}$ Peptone and $20 \mathrm{~g} / \mathrm{L}$ Dextrose) with additional $40 \mathrm{mg} / \mathrm{ml}$ geneticin (G-418 Sulphate, Gibco) only for the selection and growth of the $X$. dendrorhous mutants. The minimal medium for $S$. cerevisiae consisted of $13.4 \mathrm{~g} / \mathrm{l}$ Yeast Nitrogen Base without amino acids, 20 g/L Dextrose, $100 \mathrm{mg} / \mathrm{L}$ leucine, $40 \mathrm{mg} / \mathrm{L}$ histidine, $40 \mathrm{mg} / \mathrm{L}$ tryptophan and $40 \mathrm{mg} / \mathrm{L}$ of uracil. The same concentrations of Yeast Nitrogen Base and Dextrose were kept for the minimal medium for $X$. dendrorhous and geneticin was added to the medium for the culturing of the mutants.

\section{Construction of the E. coli strain EcCop6 and time course analysis}

The plasmid pHis8Cop6 was a kind gift of Prof. Claudia Schmidt-Dannert from University of Minnesota and it contains the cDNA sequence of the Cop6 gene from Coprinus cinereus under the control of the T7 promoter. E. coli BL21 (DE3) colonies containing pHis8Cop6 were selected on LB plates with kanamycin.

One transformed colony, EcCop6, was chosen for the time course analysis and a seed culture was started over night in LB plus kanamycin. The fresh cultures were inoculated and grown to $\mathrm{OD}_{600} 0.5$ and then $1 \mathrm{mM}$ isopropyl $\beta$-D-1-thiogalactopyranoside (IPTG) was added for the induction of expression of the Cop 6 gene. The time course was performed in duplo and consisted of six 100$\mathrm{ml}$ flasks with $10 \mathrm{ml}$ of LB medium plus kanamycin and $500 \mu \mathrm{l}$ of dodecane mixed with the internal standard, hexadecane $(68 \mu \mathrm{g} / \mathrm{ml}$ of medium), grown in a shaking incubator at $250 \mathrm{rpm}$ at $37^{\circ} \mathrm{C}$ for 48 hours. Two flasks were removed from the incubator at each sampling point 
(20, 30 and 48 hours after IPTG induction); the cultures were centrifuged at $4000 \mathrm{rpm}$ for 10 minutes, the upper dodecane layer was isolated from the medium and used for the GCMS analysis. The cell pellet was washed once with water and freeze dried to determine the cell dry weight. The same procedure was applied to $S$. cerevisiae and $X$. dendrorhous, as well.

\section{Construction of the S. cerevisiae strain ScCop6 and time course analysis}

pHis8Cop6 was used as a template for the amplification of the gene to be cloned in the p426GPD episomal vector for the expression of Cop6 in S. cerevisiae. The gene was amplified with primers flanked with the restriction sites for EcoRI and SpeI and was mutated in positions 497 and 558 to eliminate the native EcoRI sites. The fragment was then cloned in the previously digested vector and the complete construct was used to transform the S. cerevisiae wild type strain to obtain ScCop6. Positive colonies were selected on plates containing minimal medium without uracil and one positive colony was isolated and used for the time courses.

Similarly to the time course performed for EcCop6, eight $100-\mathrm{ml}$ flasks with $10 \mathrm{ml}$ medium and $500 \mu \mathrm{l}$ of dodecane and hexadecane solution were incubated at $30^{\circ} \mathrm{C}$ at $200 \mathrm{rpm}$. The four time points were chosen 24 , 48, 72 and 96 hours from the inoculation in the fresh medium and the initial $\mathrm{OD}_{600}$ of the fresh cultures was 0.05 for all the experiments.

\section{Isolation and characterization of $X$. dendrorhous mutants} The plasmids pCrtE-PSS, pCrtYB-PSS and pPR-PSS [12] were used as backbone for the creation of the new plasmids pCrtE-Cop6, pCrtYB-Cop6 and pPR-Cop6, respectively. Cop6 was amplified from the mutated gene used for the expression in $S$. cerevisiae and was flanked by the restriction sites for NheI and SalI. The fragment was then cloned in the digested vectors and used for the transformation of $X$. dendrorhous. The positive colonies were isolated for the ability to grow on selective medium with geneticin. From the transformations with pCrtECop6, pCrtYB-Cop6 and pPR-Cop6, the three new mutant strains $\triangle E$-Cop6, $\triangle Y B-$ Cop6 and XdCop6 were obtained, respectively (Figure 2). The three strains were grown at $21^{\circ} \mathrm{C}$ at $200 \mathrm{rpm}$ for the analysis of production of $\alpha$-cuprenene in time following the same conditions used for the time course for ScCop6.

\section{GC-MS analysis}

The dodecane solutions isolated from the different cultures at different time points were diluted 1:10 in ethyl acetate and run on GCMS to reveal and quantify the $\alpha$-cuprenene production. A Shimadzu GCMS-QP5000 provided with a ZB-1 ms dimethylpolysiloxane column (Phenomenex
$0.25 \mathrm{~mm}$ inner diameter, $0.25 \mu \mathrm{m}$ thickness, $15 \mathrm{~m}$ length) was used for the analysis. Two microliters of diluted dodecane were injected splitless and analyzed in total ion scan using helium as carrier gas. The GCMS program consisted of an oven initial temperature of $50^{\circ} \mathrm{C}$ with an increment of $5^{\circ} \mathrm{C} / \mathrm{min}$ up to $105^{\circ} \mathrm{C}$ and then up to $200^{\circ} \mathrm{C}$ with an increase of $30^{\circ} \mathrm{C} / \mathrm{min}$. The quantitation of the $\alpha$-cuprenene was based on the hexadecane peak which had a known concentration.

\section{Microscopy analysis and cell counting}

Aliquots of ScCop6 and XdCop6 cultures were taken after 96 hours of growth, diluted to $\mathrm{OD}_{600} 0.15$, approximately, and were then transferred to a Bürker counting chamber (Bright line, Labor Optik). The number of cells counted in a surface of $0.0025 \mathrm{~mm}^{2}$ was multiplied by $10^{4}$ and divided by the OD6 600 values of the cultures to obtain the number of cells per $\mathrm{OD}_{600}$ unit.

A Leica DM 6000B microscope provided with a 40x magnification objective and the LAS AF program was used for the visualization and to measure the size of the cells with the $10 \mu \mathrm{m}$ bar provided by the LAS AF program.

\section{Competing interest}

The authors declare that they have no competing interests.

\section{Authors' contributions}

EM conceived, designed and performed all the experiments. RS contributed to the microscopy analyses. WQ and OK supervised the research. All the authors read and approved the manuscript.

\section{Acknowledgements}

The authors wish to thank Prof. Claudia Schmidt-Dannert and Grayson Wawrzyn from University of Minnesota for providing the genes from Coprinus cinereus and for the useful discussions. The Ubbo Emmius program of the University of Groningen and the EUROCORES SYNMET programme (09-EuroSYNBIO-FP-023) have sponsored this research.

\section{Author details}

'Department of Pharmaceutical Biology, Groningen Research Institute of Pharmacy, University of Groningen, A. Deusinglaan 1, Groningen 9713AV, The Netherlands. ${ }^{2}$ Department of Technical Biochemistry, Technical University of Dortmund, Emil-Figge-Strasse 66, Dortmund 44227, Germany.

Received: 9 October 2012 Accepted: 28 January 2013

Published: 5 February 2013

\section{References}

1. Chou WK, Fanizza I, Uchiyama T, Komatsu M, Ikeda H, Cane DE: Genome mining in Streptomyces avermitilis: cloning and characterization of SAV_76, the synthase for a new sesquiterpene, avermitilol. J Am Chem Soc 2010, 132(26):8850-8851.

2. Feng $Z, K i m ~ J H$, Brady SF: Fluostatins produced by the heterologous expression of a TAR reassembled environmental DNA derived type II PKS gene cluster. J Am Chem Soc 2010, 132(34):11902-11903.

3. Berger RG: Biotechnology of flavours-the next generation. Biotechnol Lett 2009, 31(11):1651-1659.

4. Linger JG, Adney WS, Darzins A: Heterologous expression and extracellular secretion of cellulolytic enzymes by Zymomonas mobilis. Appl Environ Microbiol 2010, 76(19):6360-6369.

5. Wen F, Sun J, Zhao H: Yeast surface display of trifunctional minicellulosomes for simultaneous saccharification and fermentation of cellulose to ethanol. Appl Environ Microbiol 2010, 76(4):1251-1260. 
6. Kenny ST, Runic JN, Kaminsky W, Woods T, Babu RP, Keely CM, Blau W, O'Connor KE: Up-cycling of PET (polyethylene terephthalate) to the biodegradable plastic PHA (polyhydroxyalkanoate). Environ Sci Technol 2008, 42(20):7696-7701.

7. Westfall PJ, Pitera DJ, Lenihan JR, Eng D, Woolard FX, Regentin R, Horning T, Tsuruta H, Melis DJ, Owens A, Fickes S, Diola D, Benjamin KR, Keasling JD, Leavell MD, McPhee DJ, Renninger NS, Newman JD, Paddon CJ: Production of amorphadiene in yeast, and its conversion to dihydroartemisinic acid, precursor to the antimalarial agent artemisinin. Proc Natl Acad Sci USA 2012, 109(3):E111-8

8. Ajikumar PK, Xiao WH, Tyo KE, Wang Y, Simeon F, Leonard E, Mucha O, Phon TH, Pfeifer B, Stephanopoulos G: Isoprenoid pathway optimization for Taxol precursor overproduction in Escherichia coli. Science 2010, 330(6000):70-74.

9. Nielsen J, Keasling JD: Synergies between synthetic biology and metabolic engineering. Nat Biotechnol 2011, 29(8):693-695.

10. Schmidt I, Schewe H, Gassel S, Jin C, Buckingham J, Humbelin M, Sandmann G, Schrader J: Biotechnological production of astaxanthin with Phaffia rhodozyma/Xanthophyllomyces dendrorhous. Appl Microbiol Biotechnol 2011, 89(3):555-571.

11. Muntendam R, Melillo E, Ryden A, Kayser O: Perspectives and limits of engineering the isoprenoid metabolism in heterologous hosts. Appl Microbiol Biotechnol 2009, 84(6):1003-1019.

12. Melillo E, Muntendam R, Quax WJ, Kayser O: Heterologous expression of pentalenene synthase (PSS) from Streptomyces UC5319 in Xanthophyllomyces dendrorhous. J Biotechnol 2012, 161:302-307.

13. Agger S, Lopez-Gallego F, Schmidt-Dannert C: Diversity of sesquiterpene synthases in the basidiomycete Coprinus cinereus. Mol Microbiol 2009, 72(5):1181-1195

14. Newman JD, Marshall J, Chang M, Nowroozi F, Paradise E, Pitera D, Newman KL, Keasling JD: High-level production of amorpha-4,11-diene in a two-phase partitioning bioreactor of metabolically engineered Escherichia coli. Biotechnol Bioeng 2006, 95(4):684-91.

15. Nobbs AH, Vickerman MM, Jenkinson HF: Heterologous expression of Candida albicans cell wall-associated adhesins in Saccharomyces cerevisiae Reveals differential specificities in adherence and biofilm formation and in binding oral Streptococcus gordonii. Eukaryot Cell 2010, 9(10):1622-1634

16. Niklitschek M, Alcaino J, Barahona S, Sepulveda D, Lozano C, Carmona M, Marcoleta A, Martinez C, Lodato P, Baeza M, Cifuentes V: Genomic organization of the structural genes controlling the astaxanthin biosynthesis pathway of Xanthophyllomyces dendrorhous. Biol Res 2008, 41(1):93-108.

17. Dinh TN, Nagahisa K, Hirasawa T, Furusawa C, Shimizu H: Adaptation of Saccharomyces cerevisiae cells to high ethanol concentration and changes in fatty acid composition of membrane and cell size. PLoS One 2008, 3(7):e2623.

doi:10.1186/1475-2859-12-13

Cite this article as: Melillo et al:: Production of a-cuprenene in Xanthophyllomyces dendrorhous: a step closer to a potent terpene biofactory. Microbial Cell Factories 2013 12:13.

\section{Submit your next manuscript to BioMed Central and take full advantage of:}

- Convenient online submission

- Thorough peer review

- No space constraints or color figure charges

- Immediate publication on acceptance

- Inclusion in PubMed, CAS, Scopus and Google Scholar

- Research which is freely available for redistribution 